\title{
CLUSTER MERGERS AND NON-THERMAL PHENOMENA: A STATISTICAL MAGNETO-TURBULENT MODEL
}

\author{
R. Cassano ${ }^{1,2}$ and G. Brunettit ${ }^{2}$ \\ ${ }^{1}$ Dipartimento di Astronomia, Univ. di Bologna, Italy \\ 2 Istituto di Radioastronomia del CNR, Bologna, Italy \\ E-mail: rcassano@ira.cnr.it,brunetti@ira.cnr.it
}

\begin{abstract}
With the aim to investigate the statistical properties and the connection between thermal and nonthermal properties of the ICM in galaxy clusters, we have developed a statistical magneto-turbulent model which describes, at the same time, the evolution of the thermal and non-thermal emission from galaxy clusters. In particular, starting from the cosmological evolution of clusters, we follow cluster mergers, calculate the spectrum of the magnetosonic waves generated in the ICM during these mergers, the evolution of relativistic electrons and the resulting synchrotron and Inverse Compton spectra. We show that the broad band (radio and hard x-ray) non-thermal spectral properties of galaxy clusters can be well accounted for by our model for viable values of the parameters (here we adopt a EdS cosmology).
\end{abstract}

Key words : acceleration of particles - clusters of galaxies - radio continuum - radiation mechanism: non-thermal - turbulence - X-ray

\section{INTRODUCTION}

The most important evidences for non-thermal phenomena in galaxy clusters comes from the synchrotron radio emission diffused on Mpc scales (Radio Halos $(\mathrm{RH})$ ) observed in a growing number of massive clusters (e.g., Feretti 2003) and, more recently, from the hard X-ray tails (HXR tails) in excess of the thermal bremmstrahlung spectrum detected in a few cases (Fusco-Femiano et al. 2004). RHs are usually found in merging clusters (e.g, Schucker et al. 2001) and the detection rate of $\mathrm{RHs}$ shows a abrupt increase with increasing the X-ray luminosity and mass of the host clusters: about $30-35 \%$ of the galaxy clusters with $\mathrm{X}$-ray luminosity larger than $10^{45}$ erg $s^{-1}$ show diffuse nonthermal radio emission (Giovannini \& Feretti 2002). This observations suggest a strong connection between the existence of non-thermal components and the dynamical properties of the thermal ICM.

A promising possibility to expalain giant RHs is given by the presence of relativistic electrons reaccelerated by MHD turbulence developed during cluster mergers in the ICM (Brunetti 2003; Brunetti 2004 these proceedings). Up to now there are no theoretical works which investigate if the turbulent reacceleration is able to reproduce the statistical properties of RHs in the framework of a hierarchical scenario of cluster formation. Thus, with the aim to investigate the statistical properties and the connection between thermal and non-thermal phenomena in galaxy clusters, we have developed a statistical magneto-turbulent model. In our work, we model the formation of RHs and HXR tails in a self-consistent approach which follows, at the same

Proceedings of The 3rd Korean Astrophysics Workshop on Cosmic Rays and Magnetic Fields in Large Scale Structure time, the evolution of the thermal properties of the ICM and the triggering and evolution of the non-thermal phenomena in the framework of the magneto-turbulent re-acceleration class of models. In particular, we follow the formation and evolution of clusters of galaxies, the generation of merger-driven turbulence in the cluster volume and the acceleration and time--evolution of the relativistic particles, and the related non-thermal emission. The goals of our model are:

1 to check if cluster turbulence generated during mergers may be able to drive efficient particle acceleration processes in the ICM.

2 to investigate, in the framework of the turbulentacceleration hypotesis, if the hierarchical formation process of galaxy clusters can naturally account for the observed statistics of RHs.

In the following we describe our model and investigate the point (1); a more detailed discussion is presented in Cassano \& Brunetti 2004. The second point and the Luminosity Function of RHs expected from this model are discussed in Cassano, Brunetti, Setti, these proceedings.

\section{THE MAGNETO-TURBULENT MODEL}

\section{(a) Cluster Formation}

The evolution and formation of galaxy clusters is computed making use of a relatively simple semianalytical procedure based on hierarchical Press \& Schechter 1974 (PS) theory of cluster formation. We use the extended PS formalism developed by Lacey \& Cole (1993) which gives the probability that a parent cluster of mass $M_{1}$ at time $t_{1}$ had a progenitor of mass $M_{2} \rightarrow M_{2}+d M_{2}$ at some earlier time $t_{2}$, with 
$M_{1}>M_{2}$ and $t_{1}>t_{2}$ (e.g., Lacey \& Cole 1993, Randall, Sarazin \& Ricker 2002):

$$
\begin{aligned}
& P\left(M_{2}, t_{2} \mid M_{1}, t_{1}\right) d M_{2}=\frac{1}{\sqrt{2 \pi}} \frac{M_{1}}{M_{2}} \times \\
& \frac{\delta_{c 2}-\delta_{c 1}}{\left(\sigma_{2}^{2}-\sigma_{1}^{2}\right)^{3 / 2}}\left|\frac{d \sigma_{2}^{2}}{d M_{2}}\right| \exp \left[-\frac{\left(\delta_{c 2}-\delta_{c 1}\right)^{2}}{2\left(\sigma_{2}^{2}-\sigma_{1}^{2}\right)}\right] d M_{2},
\end{aligned}
$$

Where $\sigma(M)$ is the rms density fluctuation within a sphere of mean mass $\mathrm{M}$ and $\delta_{c}(z)$ is the critical linear overdensity for a region to collapse at redshift $\mathrm{z}$.

Adopting a suitable change of variable (M with $S \equiv$ $\sigma^{2}(M)$ and t with $\left.x \equiv \delta_{c}(t)\right)$ Eq.(1) is replaced by:

$$
K(\Delta S, \Delta x) d \Delta S=\frac{1}{\sqrt{2 \pi}} \frac{\Delta x}{(\Delta S)^{3 / 2}} \exp \left[-\frac{(\Delta x)^{2}}{2 \Delta S}\right] d \Delta S .
$$

Thus the probability that a merger with a given $\Delta S$ (i.e. $\Delta M$ ) occurs at a given time can be calculated by making use of the cumulative distribution of subclusters masses:

$$
\begin{array}{r}
\mathcal{P}(<\Delta S, \Delta x)=\int_{0}^{\Delta S} K\left(\Delta S^{\prime}, \Delta x\right) d \Delta S^{\prime}= \\
\operatorname{erfc}\left(\frac{\Delta x}{\sqrt{2 \Delta S}}\right),
\end{array}
$$

where $\operatorname{erfc}()$ is the complementary error function.

We employ a Monte Carlo procedure which selects a random number, $r$, in the range $0-1$, and determines the corresponding value of $\Delta S$ solving numerically the equation: $\mathcal{P}(<\Delta S, \Delta x)=r$. The value of $S_{2}$ of the progenitor is given by $S_{2}=S_{2}+\Delta S$ and the mass by $\sigma^{2}\left(M_{2}\right)=S_{2}$. The mass of the other subcluster is $\Delta M=M_{1}-M_{2}$. This procedure is thus iterated until the mass of the main cluster drops below a critical value or when a maximum redshift of interest is reached. An example of merger tree obtained from our procedure is illustrated in Fig.1.

\section{(b) Turbulence in Galaxy Clusters}

The turbulence in the ICM is supposed to be injected during cluster mergers. The energetics of the turbulence injected in the ICM is calibrated with the $P d V$ work done by the infalling subclusters in passing through the volume of the most massive one. This energetics is given by $E_{t} \simeq<\rho>_{I C M} v_{i}^{2} V_{t}$ where $V_{t}$ is the volume sweaped by the infalling subhalos which is estimated following standard recipes based on Ram Pressure Stripping (e.g., Fujita, Takizawa, Sarazin 2003; Cassano \& Brunetti 2004) and $v_{i}$ is the impact velocity between the two colliding subclusters. Turbulence driven by a given merger is assumed to be injected and

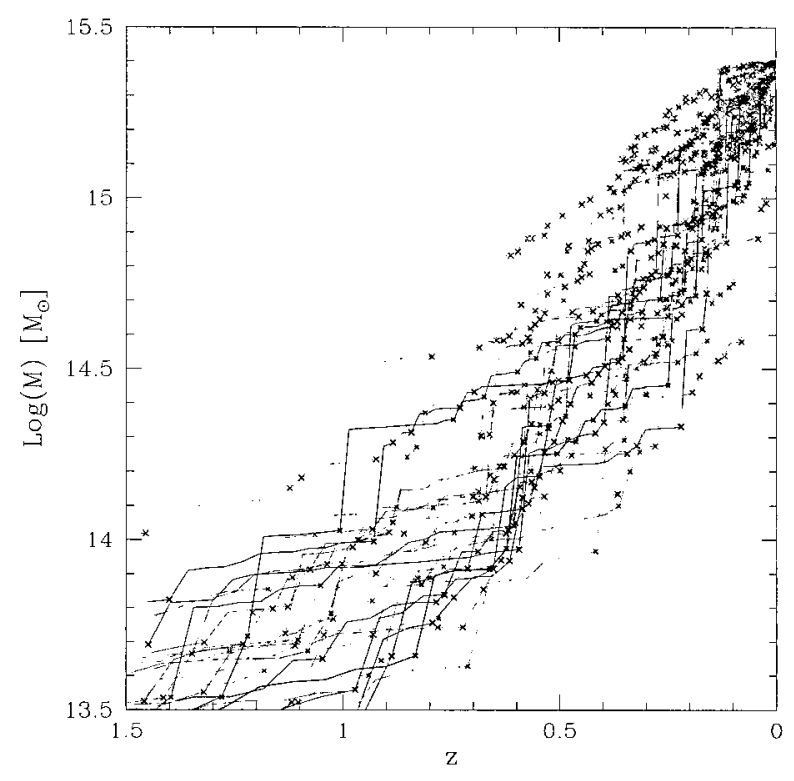

Fig. 1.- Example of Merger Trees obtained from our Monte Carlo simulations in a EdS cosmology for a cluster with a present day mass $M_{\circ}=2.5 \times 10^{15} M_{\odot}$; each cross marks a merger event with $M_{2} \geq 10^{13} M_{\odot}$

then dissipated within a crossing time, $\tau_{\text {cros }}$. We assume that a fraction $\eta_{t}$ of the energy of the turbulence developed during these mergers is channeled into fast magnetosonic waves (MS waves), $E_{M S}=\eta_{t} E_{t}$.

We use these waves since their damping rate and time evolution basically depend on the properties of

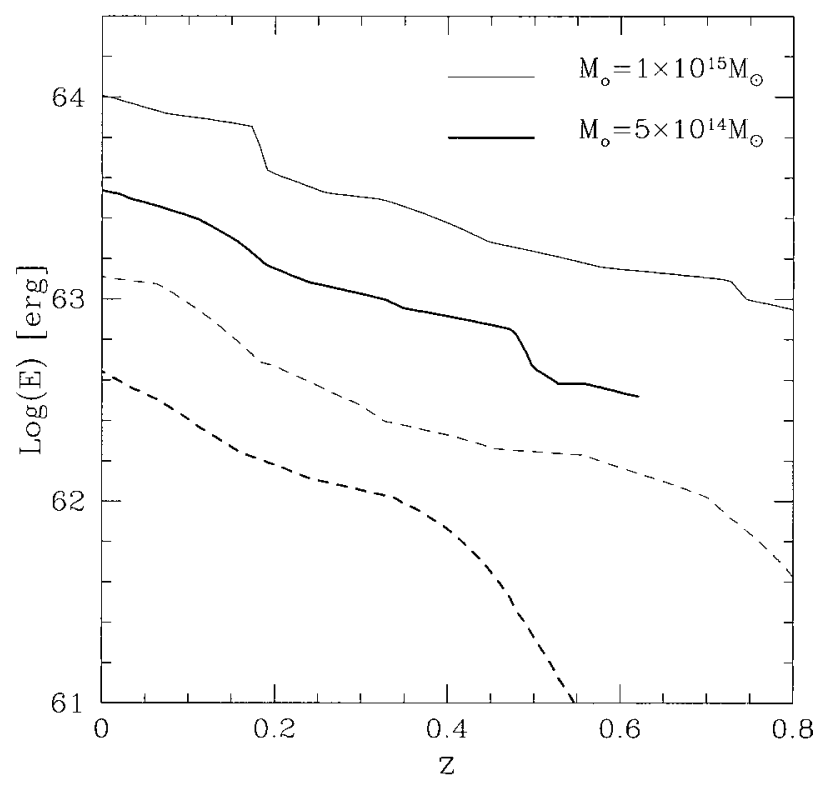

Fig. 2.- Evolution of the thermal energy (solid lines) injected in fluid turbulence (dashed lines) in typical galaxy clusters. The thin lines are for a cluster with present time mass $M_{\circ}=10^{15} M_{\odot}$ and the tick lines are for a cluster with present time mass $M_{\circ}=5 \times 10^{14} M_{\odot}$. 

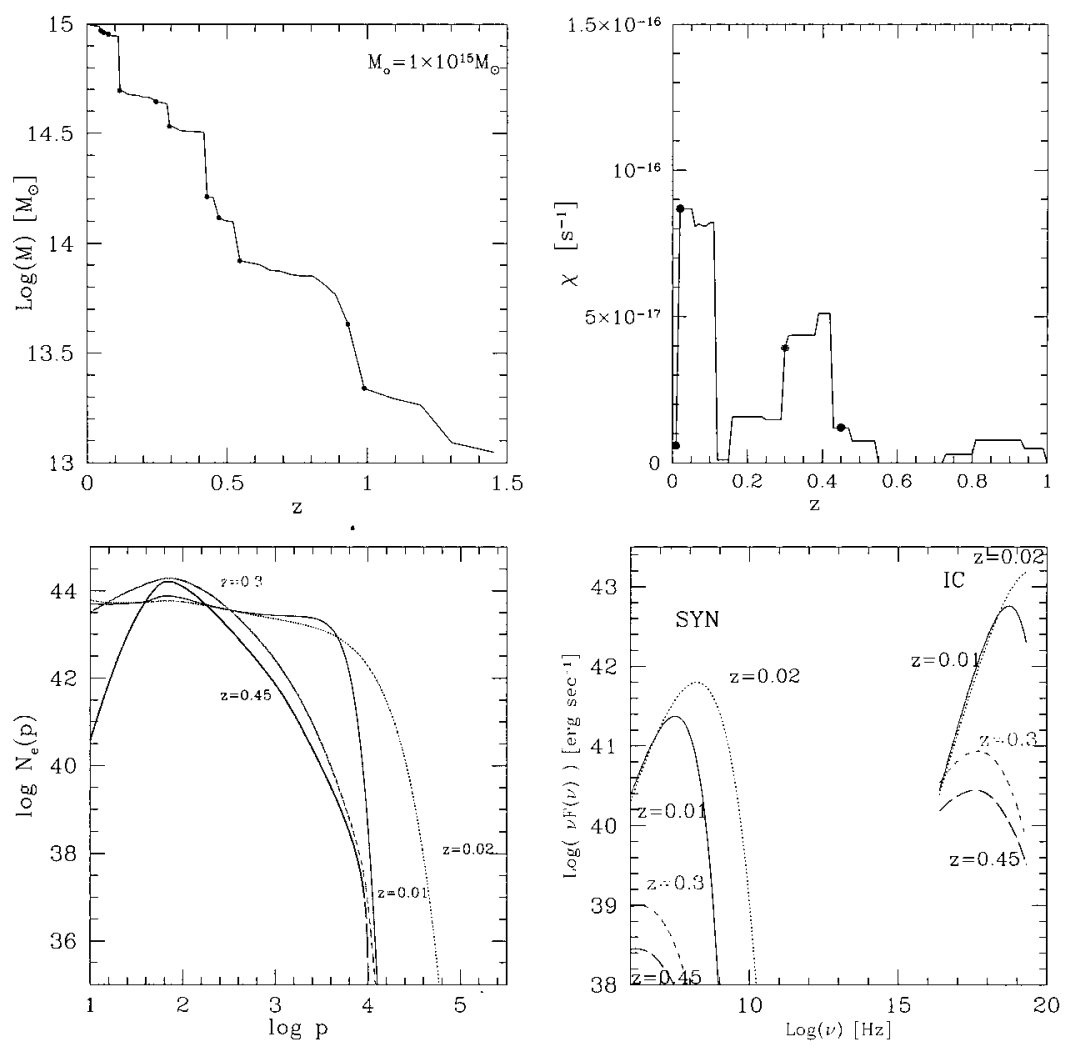

Fig. 3. - a) a singol merger tree for a cluster with present-day mass of $M_{o}=1 \times 10^{15} M_{\odot}$, each dot represents a merger event with $M_{2} \geq 10^{13} M_{\odot} ; \mathrm{b}$ ) the time-evolution (with redshift) of the electron acceleration coefficient $\left(\tau_{a c c}=\chi_{a c c}^{-1}\right)$ during the cluster formation; c) the evolution of the electron spectrum at different relevant times (see panel) in the same cluster; c) the corresponding radio (synchrotron) and hard-X ray (IC) emission. Calculations are performed assuming: $R_{H}=500 h_{50}^{-1} K p c$, $\eta_{t}=0.26, \eta_{e}=0.003$ and $\langle B\rangle=0.5 \mu \mathrm{G}$.

the thermal plasma which are provided by our merger trees for each synthetic cluster. As a relevant example, in Fig. 2 we report the cosmological evolution of the thermal energy of galaxy clusters with different masses, together with the total energy injected in form of turbulence in the ICM. The energy in form of turbulence is calculated by integrating the contributions from all the merger events experienced during the cluster life. The thermal energy of the considered clusters, calculated assuming the observed $M$ - $T$ relation (e.g., Nevalainen et al. 2000), increases from about $10^{62} \mathrm{erg}$ at $z \sim 1$ to a few $10^{64} \mathrm{erg}$ at the present epoch depending on the mass of the cluster. As it should be, we note that the energy budget injected in turbulence during cluster formation is well below the thermal energy $(\sim 15 \%$ of it), which is also in agreement with recent numerical simulations (Sunyaev et al. 2003) and with very recent observational claims (Schuecker et al. 2004). Finally, as reasonably expected, the energy injected in turbulence calculated with our approach is found to roughly scale with the thermal energy of the clusters.

Under the physical conditions in the ICM, the spectrum of MS waves injected during each merger can be estimated as (Cassano \& Brunetti 2004):

$$
W_{\mathrm{k}} \simeq \frac{I(\mathrm{k})}{\Gamma_{\mathrm{th}, \mathrm{e}}(k)}
$$

where $\Gamma_{t h, e}$ is the damping coefficient with thermal electrons and $I_{k}$ is the spectrum of the injection rate of MS waves (we assume a Kolmogorov spectrum, $\left.I(k) \propto k^{-5 / 3}\right)$. We are interested in the energy of the turbulence injected in a volume typical of a $\mathrm{RH}$ $\left(V_{H}=4 \pi R_{H}^{3} / 3\right)$. The normalization of $I(k)$ is thus given by:

$$
V_{H} \int_{k_{0}} I(k) d k \simeq \frac{\eta_{t} E_{t}}{\tau_{c r o s}},
$$

where $k_{0}$ is the wavenumber correspondent to the maximum scale which is fixed at the stripping radius for each merger event (Cassano \& Brunetti 2004).

\section{(c) Particle Acceleration}

We assume the presence of relativistic electrons in the ICM which are continuously injected by AGNs, 
Galactic Winds, and/or merger shocks. Given the calculated (Sect. b)) spectrum of MS waves and the physical conditions in the ICM (Sect. a)), we compute the time evolution of relativistic electrons at each time step by solving a Fokker-Planck equation including the electron acceleration due to MS waves:

$$
\begin{aligned}
\frac{\partial N(p, t)}{\partial t}= & \frac{\partial}{\partial p}\left[N(p, t)\left(\left|\frac{d p}{d t}\right|_{\mathrm{rad}}+\left|\frac{d p}{d t}\right|_{\mathrm{c}}-\frac{2}{p} D_{\mathrm{pp}}\right)\right] \\
& +\frac{\partial}{\partial p}\left[D_{\mathrm{pp}} \frac{\partial N(p, t)}{\partial p}\right]+Q_{e}(p, t)
\end{aligned}
$$

$(d p / d t)_{c}$ is the Coulomb losses term due to the interation with the thermal plasma (proportional to the density of the ICM) and $(d p / d t)_{\text {rad }}$ is the term of radiative losses due to the synchrotron and IC scattering off the CMB photon. For the injection rate of relativistic electrons we adopt a power law spectrum up to a maximum momentum:

$$
Q_{e}(p)=K_{e} p^{-s}
$$

We normalize the injection rate by assuming that the total energy injected in relativistic electrons during the cluster life is a fraction, $\eta_{e}$, of the total thermal energy of the cluster at $z=0$.

The electron diffusion coefficient in the momentum space due to the interaction with the MS waves (e.g., Eilek 1979; Cassano \& Brunetti 2004) is given by:

$$
D_{\mathrm{pp}}(p, t) \simeq 4.45 \pi^{2} \frac{v_{M}^{2}}{c} \frac{p^{2}}{B^{2}} \int_{k_{m i n}}^{k_{\max }} k \mathcal{W}_{k}^{B}(t) d k
$$

The acceleration time scale is $\tau_{a c c}^{-1}=\chi \simeq 2 D_{p p} / p^{2}$ and thus the systematic energy gain of electrons due to MS waves is $(d p / d t)_{a c c}=\chi p$. The coefficient of electron acceleration at redshift $\mathrm{z}$ is obtained by combining the effect due to the MS waves injected during the mergers which contribute to the turbulent spectrum at $\mathrm{z}$, one has (Cassano \& Brunetti 2004):

$$
\begin{aligned}
\chi(z) \simeq & \frac{2.23 \times 10^{-16} \eta_{t}}{\left(R_{H} / 500 \mathrm{kpc}\right)^{3}} \\
& \times \sum_{j}\left[\left(\frac{M_{1}+M_{2}}{2 \times 10^{15} M_{\odot}} \frac{2.6 \mathrm{Mpc}}{R_{1}}\right)^{3 / 2}\right. \\
& \left.\times \frac{\left(r_{s} / 500 \mathrm{kpc}\right)^{2}}{(k T / 7 \mathrm{keV})^{1 / 2}}\right]_{j}
\end{aligned}
$$

where $R_{H}$ is the radius of the $\mathrm{RH}$ and $r_{s}$ is the stripping radius of the subclumps.
In Fig.(3) we report an example of our results for a cluster with present-day mass $M_{\circ}=10^{15} M_{\odot}$. In Fig.(3a) we report the merger history (merger tree) which is obtained by using extended P\&S formalism. Given the merger tree, we calculate the turbulence injected during cluster mergers and assume that a fraction $\eta_{t}$ is channeled in MS waves $\left(\eta_{t} \simeq 0.26\right.$ is adopted in this case). The coefficient of electron-acceleration is calculated considering all the merger events which contribute to the injection of turbulence at redshift $\mathrm{z}$ in the considered cluster (Fig.(3b)). The evolution with redshift of the electron spectra (Fig. (3c)) is obtained solving the Fokker-Planck equation (Eq. (6)) which accounts for the relevant energy losses and the acceleration of the relativistic electrons due to MS waves. In Fig.(3) we assume that the energy injected in relativistic electrons during the cluster life is a fraction $\left(\eta_{e}=0.003\right)$ of the present energy of the thermal pool and calculate the synchrotron and IC emission spectra at different redshift (Fig.(3d)) from a region of $R_{H} \sim 500 \mathrm{kpc} h_{50}^{-1}$ and adopting an average magnetic field of $\langle B\rangle \simeq 0.5 \mu \mathrm{G}$.

The main goal of Fig.(3) is to show that the typical observed radio, $L_{R} \simeq 10^{40}-10^{41} \mathrm{erg} s^{-1}$ (e.g., , Feretti 2003) and hard X-ray, $L_{H X} \simeq 10^{43}-10^{44} \mathrm{erg} s^{-1}$ (e.g., , Fusco-Femiano et al. 2003) luminosities can be obtained in $M \gtrsim 10^{15} M_{\odot}$ clusters during major merger events, provided that a fraction of the cluster thermal energy (of the order of $3-5 \%$ ) is channeled into MS waves. The additional requirement is that a population of relativistic electrons to be reaccelerated is present in the ICM. We find that a total energy injected into these electrons of a few $10^{-4}-10^{-3}$ times the present energy of the thermal pool is sufficient.

\section{ACKNOWLEDGEMENTS}

G.B. and R.C. acknowledge partial support from CNR grant CNRG00CF0A.

\section{REFERENCES}

Brunetti, G. 2003, in 'Matter and Energy in Clusters of Galaxis', ASP Conf. Series, vol.301, p.349, eds. S. Bowyer and C.-Y. Hwang

Cassano, R., \& Brunetti, G. 2004, submitted to MNRAS

Eilek, J. A. 1979, ApJ, 230, 373

Feretti L. 2003, in 'Matter and Energy in Clusters of Galaxis', ASP Conf. Series, vol.301, p.143, eds. S. Bowyer and C.-Y. Hwang

Fujita, Y., Takizawa, M., \& Sarazin, C. L. 2003, ApJ, 584, 190

Fusco-Femiano, R., Orlandini, M., Brunetti, G., Feretti, L., Giovannini, G., Grandi, P., \& Setti, G. 2004, ApJ, 602, 73

Giovannini, G., Tordi, M., \& Feretti, L. 1999, NewA, 4, 141

Lacey, C., \& Cole, S. 1993, MNRAS, 262, 627 
Nevalainen, J., Markevitch, M., \& Forman, W. 2000, ApJ, 532,694

Press, W. H., \& Schechter, P. 1974, ApJ, 187, 425

Randall, S. W., Sarazin, C. L., \& Ricker, P. M. 2002, ApJ, 577,579

Schuecker, P., Böhringer, H., Reiprich, T. H., \& Feretti, L. 2001, A\&A, 378, 408

Schuecker, P., Finoguenov, A., Miniati, F., Boehringer, H., \& Briel, U. G. 2004, A\&A, submitted; astro-ph/0404132

Sunyaev, R. A., Norman, M. L., \& Bryan, G. L. 2003, Astronomy Letters, vol. 29, p. 783-790 